\title{
Clinical and therapeutic aspects of the state of shock
}

$\mathrm{S}$ hock is seen as a sequence of events initiated by an aggressive factor (hypovolumetric trauma, burns, sepsis) followed by a endocrine response (release of $\mathrm{ACTH}$, adrenaline, vasopressin) and a failure in the maintenance mechanism of homeostasis at the hemodynamic level, with a decrease in effective tissue perfusion. This leads to cellular ischemia and to the release of cytokinins (a factor in tumoral interleucinas necrosis 1,6 and 8 , nitric oxide and endothelins) which result in a systemic inflammatory process, which, if not controlled in time, becomes irreversible. The treatment itself can accentuate the inflammatory process, which is called, reperfusion lesion.

The diagnostic is clinical, instrumental and laboratorial, and the objectives of treatment are:

- to prevent the aggressive factors (a public heath measure);

- to replace the lost volume in accordance with the type of aggression;

- to monitor the endocrinal response, and before the fact to try to modulate the release of catecholamines and vasopressin;

- to the improve the effective tissue perfusion;

- to modulate the release of cytokinins and;

- to minimize the inflammatory process and reperfusion lesions.

In the replacement of volume, a hypertonic solution of sodium chloride ( $\mathrm{NaCL}$ ) $7.5 \%$ has been shown to have great potential because it:

- is low cost and rapidly portable to the aggression site;

- causes rapid hemodynamic improvement;

- improves all-over tissue edema;

- improves tissue perfusion through volumic expansion and a decrease in the endotelio edema of microcirculation;

* Full professor of the Emergency Medicine Discipline, Faculty of Medicine - University of São Paulo.
- modulates the excessive release of vasopressin and;

- indicates (suggests) a modulation of the inflammatory response

In volumetric replacement with whatever expander, we must associate vasoactive drugs if an average PA of $>$ $60 \mathrm{mHg}$ is not maintained. The next step is to monitor the patient and try to achieve a cardiac rate above 2.2 in cardiogenic shock, and above $4.0-4.5$ in sepsis and hypovolemia.

As to the mediators involved, in sepsis a bacterial endotoxin can provoke an increase in TNF and interleucinas 1,6 and 8, which in turn release nitric oxide and cause arterial vasodilation. The use of monoclonal antibodies against endotoxin or taurolidin does not lower mortality in sepsis. On the other hand, the use of the antiTNF antibody increases survival in sepsis.

The use of n-metil arginin, which reduces the release of nitric oxide, still requires more conclusive tests as to its benefits in sepsis. The inhalatory use of nitric oxide shows an improvement in the ratio perfusion-ventilation.

In the control of a patient in shock, it is important to monitor the regional flow distribution and eventual organic failures (renal and hepatic insufficiencies, mental confusion and CIUD). The lactate and the intramucosal $\mathrm{pH}$, and the enzymes and proteins of the affected organs could be useful in following the patient.

Some procedures in cardiac shock in acute myocardial infarction deserve consideration, such as angioplastia and revascularization through medication or surgery.

In conclusion, it must be stressed that despite the sophistication of the propedeutics, new medications and mechanisms in the treatment of shock, nothing substitutes the physician being constantly at the patient's side, knowing how to interpret the significance of symptoms (mental confusion, paleness, sweatiness, tachycardia, cyanosis, base stertor, PVC, etc.) for the success of the therapy. 